\title{
EL PROFESOR DE INGLÉS EN LOS TIEMPOS DE USO DE LA TECNOLOGÍA
}

\section{THE ENGLISH TEACHER IN THE TIMES OF USE OF THE TECHNOLOGY}

\author{
Heriberto González Valencia ${ }^{1}$ \\ Astrid Ramírez Valencia ${ }^{2}$ \\ German Darío Isaza Gómez ${ }^{3}$
}

END- UDFJC

\section{RESUMEN}

Este artículo busca mostrar los resultados del análisis hecho frente a la relación existente entre la tecnología y el docente inglés, considerando su efecto en la educación de este en el contexto 1 Heriberto González Valencia ORCID: https://orcid.org/0000-0001-9103-2152

Docente investigador Institución Universitaria Escuela $\mathrm{Na}$ cional del Deporte. Doctorando en Investigación en Humanidades Artes y Educación de la Universidad Castilla La Mancha, España. Miembro activo del Grupo de Investigación EDUCAR 2030 de la END. Investigador JUNIOR Colciencias.

Correos: hery77@hotmail.com heriberto.gonzalez@endeporte.edu.co

2 Astrid Ramírez Valencia ORCID: https://orcid. org/0000-0002-3025-5982

Docente Universidad Distrital Francisco José de Caldas Bogotá, Colombia, PhD. en Lenguaje y cultura, formadora de profesores de inglés durante más de 25 años. Investigadora y maestra en la Universidad Distrital Francisco José de Caldas. Correo: aramirezv@udistrital.edu.co 3 German Darío Isaza Gómez ORCID: orcid. org/0000-0001-8475-9994

Docente investigador Institución Universitaria Escuela Nacional del Deporte. Doctorando en Educación de la Universidad de la Salle, Costa Rica. Líder del grupo de investigación EDUCAR 2030 de la Institución Universitaria Escuela Nacional del Deporte. Correo: german.isaza@endeporte.edu. co. de la práctica docente, abordando la importancia de este educador en la era de la tecnología, utilizando como referencia sus reflexiones y comentarios. Respecto al tipo de investigación, se utilizó la cualitativa e interpretativa, tanto en la recolección de las muestras, así como en su el análisis interpretando las respuestas dadas por los participantes, que correspondieron a un grupo de estudiantes de práctica docente, se empleó un grupo discusión, dentro del cual se abordaron temáticas relacionadas con el uso de la tecnología, en el contexto educativo. Se concluyó que sí hay influencia de la tecnología en el ejercicio docente desarrollado por estos futuros profesores, por lo tanto, es indispensable que se generen espacios y condiciones para el uso pedagógico de estas herramientas, optimizadoras del ejercicio docente.

PALABRAS CLAVE: tecnología, docente de inglés, rol del profesor, aula de clase 


\section{ABSTRACT}

This article looks for present the results of the analysis made using a relationship between technology and the English teacher, considering the effect of technology on the education process in the future of the English language teacher in the context of pedagogical practice, taking into a count the importance of this educator in the age of technology, using as reference his reflections and comments from the previous point of view. As regards the methodology, a qualitative and interpretive investigation was developed, because both in the collection of the samples, as well as in their analysis, the answers given by the participants that corresponded to a group of pedagogical practice students were taken into account. The researcher made an interpretation of them. The high influence of technology in the teaching exercise was concluded. developed by these future teachers, to the point that it is essential that academic spaces be included within which the pedagogical management of these tools is taught, within the undergraduate programs, so it is also identified that the English teacher every day It has more relevance in making decisions related to the use of technology when they are in their pedagogical exercise.

KEY WORDS: Technology, English teacher, teachers' rol, classroom.

\section{INTRODUCCIÓN}

Pensar en la formación del docente de inglés nos lleva a considerar nuevos escenarios dentro de los cuales deberá desempeñarse, caracterizados por la virtualidad. Este cambio se ha conseguido por los aportes de las TICs (tecnologías de la información), aseguraron González, Ramírez y Salazar, (2018) la tecnología en la educación constituye uno de los mejores avances en el quehacer docente, las cuales deben hacer parte de las herramientas metodológicas, impulsadoras de otros modos de enseñanza de la lengua inglesa, pero aquí cabe preguntarnos qué tipo de docente se requiere en estas condiciones.

Adviértase que frente a la amplia gama de posibilidades ofrecidas por las plataformas virtuales generadoras de otros modos de información y conexión, que rompen con las barreras de la presencialidad, el espacio y el tiempo entre los miembros pertenecientes a determinada comunidad, se han generado otros múltiples roles del docente, llevándolo a ser: consultor, colaborador, facilitador desarrollador de contenidos, así como creador de nuevas formas de enseñar, llevándolo incluso también a ser supervisor del funcionamiento de estas herramientas. Por lo tanto, tal como lo planteo Gisbert (2002), este docente de la sociedad del conocimiento, tiene una nueva definición. La tecnología como un complemento en la educación tradicional (González, 2015).

Con este tipo de situaciones se afianza aún más la importancia del profesor de lengua extranjera, por ser quien decide cómo se debe generar la comunicación, la discusión y la presentación de los tópicos a ser tratados en la clase, teniendo en cuenta criterios tales como: los intereses de los participantes, sus necesidades de aprendizaje, edades y opiniones entre otros juicios. En este artículo se analizará el realce de la importancia del profesor de inglés en los tiempos de uso de la tecnología. Valencia, Enríquez y Agredo (2017) propusieron que el la integración de la tecnológica en la educación permite que los procesos de enseñanza y aprendizaje conviertan en un experiencia significativa.

En consecuencia, el profesor estará constantemente involucrado en un proceso de actualización, respecto al desarrollo de las nuevas formas de comunicación e información desarrolladas a través de la tecnología, manteniéndose informado sobre los usos que se le pueden dar este tipo de artefactos, buscando siempre su funcionalidad en los procesos de 
enseñanza de la lengua inglesa. Al respecto, Bates (2015), explico que el profesor hace uso de la tecnología con dos propósitos, el primero, le ayuda a desarrollar más adecuadamente su metodología, y el segundo, facilita los procesos de aprendizaje del estudiante y las investigadoras han percibido un tercer aspecto, relacionado con la innovación y la motivación hacia el aprendizaje por parte de los niños en el aula de clase.

Por lo tanto, estos avances tecnológicos se deben convertir en herramientas facilitadoras de los procesos de aprendizaje de una lengua, pero siempre teniendo en cuenta que el/la profesor/a es quien determina, el momento en que deben usarse dentro del aula de clase, la frecuencia y el modo en que debe emplearse, de tal manera que el estudiante determine el uso de las Tics para afianzar su proceso de aprendizaje de la lengua inglesa y no para lo que comúnmente lo utiliza.

Es así, como el/la profesor/a de lengua inglesa para este fin, adaptará la tecnología a los procesos pedagógicos facilitadores de su actividad docente desarrollados dentro del aula, de tal manera que la tecnología se vea como un aliado y no como un enemigo para afianzar lo anteriormente dicho, Kenski (1998) citado por Medeiros (2001), reconoció que la utilización de las TIC afecta todos los campos educativos. La educación es uno de los campos más permeados por la fenómeno de la tecnología (González, 2015, citado por Valencia, Valencia \& Enriquezia, 2019).

Con respecto esta mirada, hay que contemplar que ante los cambios siempre habrán profesores/ as de inglés que se niegan a integrar la tecnología dentro de los procesos de enseñanza de las lenguas, argumentando que les resulta muy difícil dejar de lado el uso del tablero, así como el empleo del libro, manifestando al mismo tiempo, una gran dificultad frente al manejo de la tecnología, trayendo consigo una nula aplicación de estas nuevas técnicas, en la metodología de sus clases de inglés, aunado a las condiciones tecnológicas mínimas facilitadoras de la inclusión tecnológica. Esta condición recrudece las posturas que niegan el avance de la tecnología en los tiempos actuales en los cuales está inmersa la educación.

\section{IMPORTANCIA DE LA TECNOLOGÍA}

La importancia del uso de la tecnología en el aula de clase debe solucionar en primera instancia, las necesidades de quienes están interesados en aprender un idioma, incluyéndose en los escenarios cotidianos de los jóvenes de hoy, que rompen con limitaciones identificadas en otros tiempos y que por el contrario, como lo estableció García (2014) ofrece una alternativa al profesor, quien tendrá la oportunidad de explorar nuevos recursos que están a su alcance, con los cuales será posible generar cambios en los procesos pedagógicos. En la era tecnológica, existen diversas herramientas para aprender un idioma (González-Valencia, IsazaGómez, Idarraga y Rodríguez-Villaquiran, 2020). González y Gertrudix (2020) argumentaron que la educación siempre está en proceso de evolución, y los educadores siguen ese ritmo y tratan de estar a la par con todos los cambios que esta propone.

Con esto se quiere establecer que la tecnología debe convertirse en una herramienta elemento facilitadora del proceso de enseñanza que el profesor de inglés, debe aprovechar, sin convertirlo en el único fin del ejercicio docente del profesor, sino integrándose al contexto de los jóvenes como un líder o experto, en apoyo de en las decisiones pedagógicas que lo requieran.

Lo anterior lo reafirmo Aguilar (2012), manifestando que en la actualidad estamos siendo abocados a vivir en una época, donde el cúmulo de información produce un aceleramiento de interacciones y dinámicas educativas, convulsionadas por los múltiples cambios 
tecnológicos, ocurridos de manera rápida, por lo tanto, el profesor debe tener experticia para encaminar la selección adecuada frente al cumulo agobiante de información existente en la red.

Otra consideración se relaciona con el uso de la tecnología frente a la comunicación y a la relación entre los miembros de las distintas comunidades, creando incluso otros lenguajes que hasta hace poco eran desconocidos, o tenían otro significado diferente al actual, tal es el caso por ejemplo de los emoticones, que han transformado su representación interpretativa, generando otros modos de uso del lenguaje, trayendo consigo también nuevos cambios en los modos de comunicar, en esta línea argumentativa, Diker (2008) aclaro que la escuela ya no es la única fuente de información, adquisición y uso del conocimiento. Una evidencia de ello se presenta a través de la enseñanza a distancia, la cual usualmente está organizada en módulos virtuales que promueven los encuentros entre distintos miembros de una comunidad, sin compartir un espacio físico, pero si trazando un propósito de comunicación, interacción y aprendizaje divergente. Colmenares y Barroso (2014), aclararon frente a esta situación que las Tics han generado otros modos de aprendizaje, así como otro tipo de interacción y comunicación, con una filosofía de trabajo también distinta, entre los integrantes de distintos grupos.

Aquí se reconoce que mediante la implementación de este tipo de comunicación y de enseñanza, se genera un ambiente dentro del cual el contacto directo con las personas no es relevante, prefiriendo en muchos casos establecer interacciones no mediadas por el contacto físico entre los miembros de la comunidad, pero, desvirtúan el valor emotivo de la comunicación, pues es innegable la importancia de la reciprocidad existente en el contacto con el otro, ya sea por la mirada, el gesto, la proxemia ocasionando en algunos casos la falacia entre lo que se dice y realmente se transmite (Ong, 1982).

Esta situación, ha provocado situaciones que rompen con los cánones de la comunicación, prefiriendo la comunicación virtual, individual, en algunos casos ha traído como consecuencia que las personas prefieran comunicarse por estos medios tecnológicos, y no de manera física con los miembros que hacen parte de su comunidad, corriendo el riesgo de perder la habilidad de la oralidad, o de pronto cambiándola por las nuevas circunstancias de la interactividad, que desconocen al otro como parte de un grupo, llevando incluso, a una crisis existencial, pues recordemos que somos seres sociales desde nuestro vientre y estos medios nos están mutilando esta condición de nuestro ser, poco a poco.

Por consiguiente, es innegable que a pesar de la ventaja ofrecida por la comunicación mediada por la tecnología que rompe con las barreras del espacio, se está generando modos de información mucho más ajustables a las necesidades de las personas, para quienes en algunos casos es más fácil establecer enlaces usando la tecnología, y no de manera directa especialmente por las distancias y la comodidad ofrecida por esta clase de intercambio de opinión, pero se está corriendo el riesgo se transmutar la habilidad oral, pues existe la tendencia a evitar los encuentros reales con otros, por preferir a cuatro mil amigos virtuales.

Por esto, el docente de inglés está obligado a entrar en esta nueva dinámica de comunicación y de creación de conciencia respecto a estas dificultades, con el propósito de interactuar de manera asertiva con sus estudiantes, para empezar a adentrarse en el mundo de las nuevas formas de enseñar; por lo cual es necesario tener un sentido de criticidad sin abandonar su condición humana, manteniendo una constante reflexión frente a las implicaciones 
que tiene el uso de la tecnología, orientando a sus estudiantes en el uso responsable de las mismas .

Esta idea es reforzada por Zamora (2011), quien explico como la presencia digital en la sociedad y las aulas han producido un nuevo campo de distorsión, un "nuevo ruido en la relación pedagógica" llevando a los docentes a pensar en la necesidad de involucrarnos en estas nuevas formas de enseñar, cuya práctica, predomina en estos tiempos modernos.

Este cúmulo de ideas nos lleva a pensar sobre las ventajas ofrecidas por la tecnología en los procesos de enseñanza llevados a cabo por el profesor, quien al utilizar este tipo de recursos logra captar la atención de sus estudiantes, especialmente porque puede conectarse con la realidad vivida por ellos, logrando así su participación activa. Guerreo \& Faro (2012) explico cómo los recursos tecnológicos generan buenos niveles de eficacia en la enseñanza $y$ aseguran el éxito en el aprendizaje de los alumnos.

La UNESCO (1998) explico como el rápido avance de las TIC ha cambiado la forma de elaboración, adquisición y transmisión de conocimientos, razón por la cual, el docente debe ser un filtro en la búsqueda de un conocimientos veraz y científico. Para esto, se requiere un perfil y como lo plantearon Bozu \& Canto (2009), el docente debe ser flexible y variante, capaz de adecuarse a los frecuentes cambios de nuestra actual sociedad.

De otro lado, el profesor de inglés también se convertirá en un identificador de problemas y situaciones de tensión dentro del contexto escolar, de tal manera que, apoyado en el desarrollo de las tecnologías, pueda dar solución a las problemáticas reconocidas, generando al mismo tiempo otras formas de entender y pensar en las interrelaciones de los jóvenes en el contexto del aula, con miras a generar un ambiente de paz y sana convivencia.

Así las cosas, el docente tendrá que hacer la transición de los modos tradicionales de enseñar para vincularse con las nuevas dinámicas impuestas por el uso de las tecnologías en el contexto escolar. Al respecto, Granados (2015), establecio que "el uso de las TIC supone romper con los medios tradicionales, pizarras, lapiceros, libretas, etc. $Y$ dan paso a la función docente, basada en la necesidad de formarse y actualizar sus métodos, en función de los requerimientos actuales." Es así como el docente, nunca perderá su rol de acompañamiento, porque las Tics como herramientas facilitan el trabajo del docente, cobrando vigencia su papel vitalicio en la formación de la sociedad.

Aquí cobra un papel importante el conocimiento pedagógico del docente definido por Cabero, Marín \& Castaño (2015) como las actividades pedagógicas que un docente podría utilizar, así como, los procesos y prácticas de métodos de enseñanza, al igual que la forma en que estos se relacionan con el desempeño laboral del educador.

Frente a este panorama, el papel del profesor recobra protagonismo, por ser el actor tomador de decisiones, y el directo responsable de las acciones emprendidas dentro del aula, de tal manera que sus decisiones pueden romper los cánones establecidos, dando paso a nuevas formas de desarrollar el saber que posee, basado en sus experiencias, sus intuiciones, y en los demás elementos inherentes a la escuela.

En este sentido, se necesita de un docente de mente abierta, con capacidad de adaptación frente a los grandes cambios suscitados a partir de las experiencias y el contacto con estas nuevas tecnologías, con habilidad para enfrentar dificultades, buscando alternativas a los obstáculos; con el propósito de superarlos, mediante la puesta en marcha de acciones generadoras de transiciones y ajustes, acordes 
a las novedades presentadas dentro o fuera del aula de clase, dispuesto a actualizarse de ser necesario e indispensable, para lograr un mejor desempeño en sus clases de inglés, siendo sensible a la condición humana que lo rodea retadora en la formulación de cambios.

Es innegable que el constante avance de la tecnología ha hecho innovar y reinventar el quehacer profesional de más de una ocupación y labor, siendo la docencia una de las que más ha necesitado adaptarse a estas nuevas tecnologías, por esto es fundamental que el docente de inglés busque estar siempre abierto a la idea de implementar y convertir las Tics, en herramientas de uso común en sus clases. Carneiro, Toscano y Díaz (sf) determinaron que los cambios acelerados de la sociedad de la información, implican nuevos desafíos para la educación y por lo tanto son generadoras de retos en los profesores.

\section{CONSIDERACIONES DEL USO DE LA TECNOLOGÍA EN EL AULA DE CLASE}

La idea de cambiar un paradigma instaurado en el pensar de la sociedad puede ser utópica, pero no está nada lejos de ser una realidad en el contexto de la enseñanza de las lenguas, porque él ahora está quebrantado la linealidad y se está empezando a sentir el efecto de la evolución de la enseñanza, con el uso de las nuevas tecnologías.

La misión de las nuevas generaciones de docentes, entonces es, continuar desarrollando este pensamiento, pues la enseñanza, como el ser humano, deben estar dispuestos al cambio y a la adaptación, en aras de satisfacer las condiciones de la sociedad requerida, pero, en nuestro caso Colombia, de acuerdo a las políticas gubernamentales y al escaso apoyo económico en el sector educativo no se cuenta con los servicios mínimos en muchas instituciones, así como debido a su posición geográfica algunos departamentos o municipios alejados de la capital, no cuentan con las condiciones mínimas requeridas para estar acordes a las exigencias de la tecnología, esto hace que su desarrollo sea lento.

Se puede reiterar que a pesar de que las herramientas cambian, el rol del profesor como facilitador no varía, pues si bien su trabajo se transforma, su presencia en el aula (ya sea de forma presencial o virtual) es esencial, pues es quien guía y ayuda a resolver dudas garantizando que los estudiantes desarrollen adecuadamente su proceso de aprendizaje así su rol se enriquece inspirando, aclarando, compartiendo y participando activamente, en el aula y en la vida de sus estudiantes.

Cabero (2007) señalo como las nuevas tecnologías han promovido el uso de diferentes metodologías, generando cambios en el campo de la didáctica, esta situación ha convertido al docente en un creador de nuevas herramientas posibilitadoras del análisis, de la reflexión y del estudio, tanto de la educación, como de la tecnología.

Así las cosas, el docente debe estar en capacidad de orientar a cada estudiante, tomando en cuenta sus características individuales, considerando siempre la diferencia existente entre ellos, evitando concebir la educación como un proceso masivo, amorfo y sin derecho a la diferencia, siendo incluyente en todos sus aspectos.

\section{¿LA CAPACITACIÓN DOCENTE EN TECNOLOGÍA, ES SUFICIENTE PARA PROPICIAR CAMBIOS?}

Mestres (2008) determinó que el docente necesita estructurar su función educativa, teniendo definido el modo en que los estudiantes desarrollan competencias cognitivas y la forma en que son capaces de identificar su función, dentro de los contextos en los cuales se desempeña el estudiante, de tal manera que 
la tecnología le permita acceder de manera práctica al conocimiento, generándole otras formas de pensar y entender el mundo que lo rodea.

El docente actual en formación que esté dispuesto, en un futuro a implementar metodologías que satisfagan las necesidades educativas, requerirá de una formación adecuada que les permita estar a la vanguardia de las herramientas y soluciones digitales, no solo ofrecidas por el sistema educativo, sino halladas el mundo de las TIC, las cuales cada vez más van evolucionando para ser más accesibles, eficientes y veloces.

Por tal razón, en la formación educativa se deberá poner atención a dichos procesos tecnológicos cada vez más necesarios en un docente, no solo para mejorar los métodos de enseñanza o complementarlos, sino para mantener un nivel de adecuación y ajuste permanente al desarrollo de sus clases, o a su desempeño frente a los alumnos, o, por el contrario, respecto al entorno donde desarrolla su actividad docente, exigiéndolo mantenerse actualizado frecuentemente. Fernández (2001) estableció que la presencia de las nuevas tecnologías implica una reflexión en busca de mejoras educativas.

Por lo tanto, el rol del docente de inglés se transforma implementando nuevas técnicas, convirtiéndolo en un facilitador de los procesos desarrollados en el aula de clase, utilizando las tecnologías de la información y la comunicación.

Estas herramientas efectúan un cambio favorable a la enseñanza, abandonando la visión de la "transmisión de conocimientos establecidos", para empezar a emprender la búsqueda de una acción pedagógica transformadora, basada en la experimentación y la puesta en marcha de ejercicios pedagógicos, acompañados de la tecnología.
De esta forma, se demuestra que la tecnología es una herramienta facilitadora de la interconexión, y del contacto de los rasgos culturales de otras lenguas, generando espacios multiculturales, de civilizaciones inherentes a la lengua, convirtiendo así el aprendizaje del idioma, en una acción significativa, para quien la está adquiriendo y descubriendo.

Luego, el hecho de tener múltiples recursos tecnológicos al alcance del profesor, tales como, el tablero electrónico, los computadores portátiles, entre muchos otros elementos; no garantiza el uso de la tecnología dentro del aula de clase, porque si los profesores no son visionarios de la utilización de dichos recursos, los estudiantes no tendrán la oportunidad de acceder al conocimiento de forma dinámica ofrecida por la tecnología.

En consecuencia, es crucial que en la educación del docente se incluyan y se implementen las competencias tecnológicas, para que los futuros docentes sean conscientes de las ventajas de manejar estas herramientas de manera adecuada promotoras de la mejora del ejercicio docente. Escudero (1992) establecio la necesidad de integrar las nuevas tecnologías en los programas educativos, con el propósito de hacer un uso pedagógico de las mismas.

Aunque, no se puede desconocer que dentro de la red existente mucha información la cual no siempre es útil, el profesor de inglés debe orientar y dirigir a sus estudiantes a aprender a seleccionar la información, dentro de la multiplicidad de información existente, de tal manera, que escoja realmente lo pertinente e importante para sus intereses; tampoco, podemos dejar de lado, la utilidad de la tecnología en el campo de la educación, por ello los estudiantes deberán darle un uso adecuado para obtener muchos más beneficios en sus procesos de aprendizaje, razón por la cual es inevitable la inclusión de las TIC en el plan de estudios de formadores de docentes. 


\section{METODOLOGÍA}

El estudio aquí presentado busca responder los siguientes interrogantes: 1) ¿Qué características debería tener el docente de inglés inmerso en el mundo de la tecnología? 2) ¿De qué modo el futuro profesor de inglés utiliza la tecnología, en sus clases de inglés?

Para responder a estos interrogantes, se analizó la influencia de la tecnología, en el proceso de formación de futuro profesor de inglés en el contexto de la práctica docente, tomando como referencia sus reflexiones y comentarios.

\section{CONSIDERACIONES METODOLÓGICAS Y POBLACIÓN}

Para esta investigación se aplicó un enfoque cualitativo, descriptivo e interpretativo aplicado en el estudio de un grupo de diez estudiantes de un programa de licenciatura en inglés, que hubiesen empleado la tecnología durante el desarrollo de su práctica docente, cuyas edades oscilaban entre los 20 y 25 años de edad, dentro de los cuales seis eran hombre y cuatro mujeres.

\section{INSTRUMENTOS}

Dentro de la metodología cualitativa se aplicó una entrevista semiestructurada para acceder a la información acerca de un aspecto particular que se quería indagar, Riba (2014) la definio como la descripción de un fenómeno social educativo. Las preguntas incluidas en la entrevista fueron validadas por cuatro expertos, cuyos conceptos avalaron en un $80 \%$ las preguntas abiertas formuladas dentro de las cuales se buscaba indagar acerca del impacto de la tecnología en la formación de los docentes de inglés.

\section{DISCUSIÓN Y RESULTADOS}

Siguiendo los autores previamente mencionados en este artículo sobre los nuevos escenarios de la enseñanza de inglés influenciados por la tecnología, se denota el gran peso que este tipo de herramientas ha tenido en el desempeño de este profesor.

A fin de dar respuesta al objetivo planteado en esta investigación el cual buscó conocer el grado de influencia de la tecnología en el proceso de formación del futuro profesor de inglés, se puede enunciar que los datos ofrecidos por los entrevistados verifican los supuestos planteados previamente. En ellos se deja entrever aseveraciones señaladas por los teóricos mencionados de forma general en relación con la importancia de la tecnología en la práctica docente del profesor de inglés.

Del mismo modo, es pertinente comprobar la importancia que el profesor ha tenido en el contexto ya mencionado. En relación con esta mirada, los análisis teóricos hechos por autores tales como Granados (2015) y García (2014), reconocieron que este estudio ha ido un poco más lejos, frente al tratamiento de esta temática.

De manera particular algunas de las consideraciones más destacadas sobre la influencia de la tecnología en el ejercicio pedagógico del futuro docente de inglés, de acuerdo a la opinión de los entrevistados, se destacaron entre otras las que a continuación se mencionan:

[...] El cambio de metodología en la enseñanza es un claro ejemplo de cómo todo va avanzando, no necesariamente por la tecnología que se vaya implementando, sino por la cantidad $y$ calidad de herramientas. Que se han ido añadiendo a la estructura de la educación [...]

Los participantes además resaltaron la facilidad de promover la renovación cuando el docente usa herramientas tecnológicas, de manera general reconocieron la efectividad de la comunicación entre los miembros de una comunidad, cuando se empezaron a usar los medios tecnológicos, dejando atrás la comunicación unidireccional 
y presencial de otros tiempos, para dar paso a niveles de comunicación, que no implican el contacto directo entre quienes hablaban.

Para ratificar los argumentos hasta aquí señalados, se presentan aleatoriamente solamente tres ejemplos, extraídos de las entrevistas:

\section{[...] las cosas son diferentes a lo que} ya era hace años, la tecnología permite que los centros educativos ofrecen sus servicios de manera masas y con bajos costos, por las nuevas maneras de comunicación existentes. [...]

[...] hay nuevos elementos que son grandes herramientas para desarrollar metodologías innovadoras [...]

[...] Hoy en día existen muchas más posibilidades a la hora de llevar a cabo una clase manifestados por ejemplo en las aulas virtuales y espacios digitales de discusión, donde los estudiantes pueden no solo adquirir conocimiento si no también compartirlo, logrando así un aprendizaje más personalizado. [...]

Por otro lado, en relación con la importancia del futuro profesor de inglés en la era de la tecnología, se destacó la trascendencia de su rol, reconociendo que sigue siendo la persona tomadora de decisiones, quien tiene muchas más funciones en la actualidad que hace algún tiempo atrás, lo que lo induce a ser imprescindible para el buen desarrollo de las clases, esto se evidencia en algunos de los siguientes apartes sacados de las entrevistas:

[...] el correcto proceso de aprendizaje, no depende de las herramientas ni de la forma de aprender, sino de cómo las utilice el profesor para obtener un mayor beneficio. [...]
[...] A pesar de que en estos tiempos cualquier persona puede educarse en cuanto a una lengua simplemente por internet si así lo quisiera, queremos rescatar el rol y la necesidad del docente en las aulas presenciales, sin dejar de lado la tecnología, podemos observar un cambio en la forma de educar sin embargo es el docente quien adapta y decide en la clase. [...]

[...] La tecnología en las aulas debe ser usada adecuadamente por parte de los profesores de lengua quienes toman las decisiones pedagógicas que faciliten el desarrollo de las clases. [...]

Se corrobora la mirada de los teóricos que determinan como los profesores exploran los recursos ofrecidos por los medios tecnológicos, para luego emplearlos en su contexto escolar de tal manera que se ven reflejados en la acción educativa del docente; por lo tanto, es este profesor quien toma las decisiones sobre qué y cómo enseñar la lengua basado en sus conocimientos didácticos, y en su experiencia, cobrando mayor relevancia la función del docente dentro de la escuela, por ser en quien cae la mayor responsabilidad de las acciones pedagógicas llevadas a cabo dentro del aula de clase.

Ahora bien, para alcanzar este propósito, como lo define Granados (2015) es importante la formación y actualización del docente, por lo cual se precisa que su proceso de educación esté acorde a los requerimientos del mundo actual, determinado por los avances tecnológicos que jamás se detienen. En opinión de los entrevistados, esto se evidencia cuando afirman: 
[...] Ante la necesidad de enfrentar un mundo "tecnológico" los maestros deben prepararse para poder compartir con sus estudiantes estas competencias tan necesarias en estos tiempos. [...]

[...] existe la necesidad de tener nuevas habilidades y conocimientos que le permitan al docente de inglés hacer uso de este tipo de herramientas dentro del aula de clase, por lo cual debe capacitarse. [...]

[...] es necesario que los docentes de inglés se encuentren dispuestos a la continua actualización del conocimiento en relación con las tecnologías de la información y la comunicación, [...]

Finalmente, se precisa de un docente de inglés dispuesto al cambio en Marín \& Castaño (2015) quienes resaltaron la trascendencia del conocimiento pedagógico del profesor requiriendo de una continua actualización, así como la inclusión de espacios de formación en tecnología, dentro de los programas que preparan a los docentes de inglés.

Días Barriga (s.f.) resalto la relevancia de llevar la tecnología al aula de clase, por lo cual es imprescindible poseer este conocimiento por parte del docente de inglés. Algunas ideas de los encuestados al respecto, fueron determinadas por las opiniones plasmadas en las entrevistas, en los siguientes apartados:

[...] La formación actual de los docentes debe ofrecer espacios donde el profesor aprenda a darle un uso pedagógico a la tecnología, de lo contrario el profesor deberá actualizarse. [...]

[...] Nuestra pedagogía, deberá estar apoyada por el uso de la tecnología por lo cual necesitamos poseer este conocimiento, para estar en capacidad de usar didácticas basadas en la tecnología,

\begin{abstract}
especialmente en esta era digital
globalizada. [...]

[...] también existen páginas donde los profesores que no sepan acerca del uso de las Tics pueden aprender. Principalmente se puede mencionar YouTube, pues allí se encuentran gran cantidad de videos donde se puede encontrar toda la información necesaria para aprender a usar adecuadamente las Tics. [...]
\end{abstract}

Para terminar, se determina la necesidad latente de involucrar a los docentes de inglés en la aplicación de herramientas tecnológicas, facilitadoras y enriquecedoras de su proceso de enseñanza, y en su formación la que nunca termina.

\section{CONCLUSIONES}

Una vez definidos los resultados alcanzados en el desarrollo de la presente investigación, surgen nuevos interrogantes a ser alcanzados en próximos estudios determinados por la necesidad de conocer, por ejemplo: ¿cuáles son las responsabilidades de los programas de formación de docentes de inglés, en relación con el manejo de las Tics?

¿Hasta qué punto el conocimiento sobre la aplicación de la tecnología, se está incluyendo en los programas que forman docentes de inglés?

¿Existe algún estudio que mida el conocimiento sobre tecnología, que tienen los futuros profesores de inglés?

A pesar de ello, el presente estudio nos permitió en primera instancia afianzar la necesidad de conocer tanto las herramientas tecnológicas, como su aplicación pedagógica en la enseñanza de la lengua, esto porque es innegable el alcance que este tipo de instrumentos tiene en los procesos de enseñanza de lengua, llevado a cabo por el profesor, hasta llegar a convertirse 
en elementos facilitadores de los procesos de aprendizaje de lengua, por parte del estudiante.

Por otro lado, a pesar de que muchos estudiantes que se encuentran cursando los programas de licenciatura de inglés son jóvenes, aunque reconocen la importancia de la tecnología, también aceptan el hecho que existen muchas circunstancias que les impide hacer uso y aplicación de las mismas dentro de sus clases, especialmente cuando se encuentran desarrollando su práctica docente por dos razones, la primera de ellas, porque no hay una logística dentro de los contextos escolares donde hacen su práctica, que posibilite el uso de estos elementos.

La segunda razón, se debe a que muchos de ellos desconocen la información relacionada con la aplicación pedagógica de la tecnología, motivo que los induce a no utilizar este tipo de herramientas, dentro de la clase de manera permanente.

Frente a esto, surge la necesidad sentida de incluir espacios de formación dentro de los programas de licenciatura en inglés, en los cuales se dediquen a formar docentes capaces de utilizar la tecnología, dándole una importante trascendencia como herramienta, para su uso pedagógico, de tal manera, que su praxis pedagógica se vuelva innovadora e interesante, para quienes están aprendiendo la lengua extranjera.

Esto se traduce en que un alto porcentaje de ellos aún mantienen un enfoque tradicional en el desarrollo de sus clases, trayendo consigo al mismo tiempo que no exista una sincronización entre la realidad vivida por los estudiantes en la escuela y las actividades organizadas por el profesor de inglés, como consecuencia y muchos de los estudiantes en las escuelas, se sienten desmotivados, para recibir el conocimiento relacionado con el aprendizaje de la lengua.
De otro lado, es necesario la implementación real de la tecnología dentro del aula de clase pues como se planteó que no basta con tener al alcance diversos dispositivos, si se les está dando el mismo uso tradicional de otros elementos utilizados por el profesor en otros tiempos, tal es el caso del tablero electrónico, no basta con tenerlo en el aula de clase, sino se le da un uso apropiado, sino por el contrario, si solamente se emplea para escribir, tal como se hacía con el tablero tradicional.

Es necesario repensar la enseñanza de la lengua extranjera inglesa, por las demandas de la generación actual, de tal manera que el profesor esté capacitado para actuar en este nuevo contexto de manera adecuada, por lo cual necesita ser competente tanto en el uso, como en la aplicación pedagógica de las tecnologías.

Finalmente, se reconoce que el docente de inglés tiene una gran oportunidad al utilizar las herramientas tecnológicas que le podrían potencializar el interés y compromiso de los estudiantes para acceder al conocimiento de la lengua.

\section{REFERENCIAS BIBLIOGRÁFICAS}

Aguilar, M. (2012). Aprendizaje y tecnologías de información y comunicación: Hacia nuevos escenarios educativos. Revista Latinoamericana de Ciencias Sociales, Niñez y Juventud, 10

Bates, T. (2015). Teaching in a Digital Age. Disponible: http://goo.gl/NAvukU

Biggs, J. (2006). Calidad de aprendizaje universitario. Madrid: Narcea.

Bozu, Z., \& Canto, P. J. (2009). El profesorado universitario en la sociedad del conocimiento: competencias profesionales docente. Revista de Formación e Innovación Educativa Universitaria, 2(2). 
Cabero Almenara, J., Marín Díaz, V., \& Castaño Garrido, C. (2015). Validación de la aplicación del modelo TPACK para la formación del profesorado en TIC. @tic. Revista d'innovació educativa, 14. Doi: http://doi.org/10.7203/attic.14.4001

Cabero, J. (2004). Formación del profesorado en TIC. El gran caballo de batalla. Comunicación y Pedagogía, 195.

Cabero, J. (2007). Las necesidades de las TIC en el ámbito educativo: oportunidades, riesgos y necesidades. Tecnología y Comunicación Educativa, 21.

Cabero, J. (2008). La formación del profesorado para el manejo de las TIC en los procesos de enseñanza/ aprendizaje. En F. Martínez (coord.). Incorporación de las TIC en los programas académicos de las Universidades Estatales Costarricenses. (pp. 55-68). Murcia, Diego Marín.

Cabero-Almenara, J., \& Díaz, V. M. (2014). Miradas sobre la formación del profesorado en tecnologías de información y comunicación (TIC). En!@ ce: Revista Venezolana de Información, Tecnología y Conocimiento, 11.

Carneiro, Toscano \& Díaz (sf). Los desafíos de las TIC para el cambio educativo. Madrid: Fundación Santillana.

Colmenares, L. y Barroso, J. (2014). Tipos de aprendizajes emergentes bajo la influencia de la Web 2.0. Revista Internacional de Investigación e Innovación educativa. (IJERI) 1.

Díaz- Barriga, F. (sf). La innovación en la enseñanza soportada en TIC. Una mirada al futuro desde las condiciones actuales. Recuperado de http://www. oei.es/tic/santillana/Barriga.pdf Díaz-
Barriga, F. (2013). TIC en el trabajo del aula. Impacto en la planeación didáctica. Revista Iberoamericana de Educación Superior, 4(10), 3-21. Doi: 10.1016/ S2007-2872(13)71921-8

Diker, G. (2008). Autoridad y Transmisión: Algunas notas teóricas para re-pensar la educación. Revista Educación y Humanismo, 15

Escudero, J. M. (1992). La integración escolar de las nuevas tecnologías de la información. España: Infodidac 21

Fernández Batanero, J. M., \& Rodríguez Martín, A. (2017). TIC y diversidad funcional: conocimiento del profesorado. EJIHPE.

Fernandez M. (2001). La aplicación de las nuevas tecnologías en la educación. Tendencias Pedagógicas, 6. Recuperado de: https://revistas.uam. es/tendenciaspedagogicas/article/ view/1811

Fernández-Batanero, J.M., \& Bermejo, B. (2012). Actitudes docentes hacia las TIC en centros de buenas prácticas educativas con orientación inclusiva. Enseñanza \& Teaching, 30(1) doi: https://doi. org/10.30552/ejihpe. v7i3.204

García González, Enrique. (2014). Morín, Edgar: La nueva realidad de la enseñanza. México: Trillas

González-Valencia, H. y Gértrudix-Barrio, F. (2020). Entornos virtuales en el ambiente escolar para el desarrollo de la competencia científica. En F. Gértrudix-Barrio y B. Rivas-Rebaque (eds.), Nuevas Alfabetizaciones. La competencia científicotecnológica en el ámbito educativo (107-120). Ed. Dykinson. ISBN: 978-84-1377-229-5. http://www.englishatuniversity.com/files/ 
libro-espana-felipe-dic-2020.pdf

González, H. (2015). La integración de la tecnología como herramienta significativa en la enseñanza del inglés como lengua extranjera. Revista Horizontes Pedagógicos. 17(1), 53-66. https://horizontespedagogicos.ibero. edu.co/article/view/17105/693

González, H., Ramírez, A., and Salazar, P. (2018). Las TIC en el mejoramiento de las competencias en lengua extranjera de los profesores de inglés. Editorial USC, Cali - Colombia. ISBN: 978-9585522-54-1 e-ISBN 978-958-5522-55-8 Recuperado de: https://repository.usc. edu.co/bitstream/20.500.12421/383/1/ Libro

González-Valencia, H. Isaza-Gómez, G.D. Idarraga, M.A. y Rodríguez-Villaquiran, M. (2020). Integrating the virtual platform memrise as a teaching tool for english vocabulary in foreign language students of second semester at a university. Revista Latinoamericana de Estudios Educativos, 16 (1), 259-284. DOI: 10.17151/rlee.2020.16.1.12

Gisbert, M. (2002) El nuevo rol del profesor en entornos tecnológicos, Acción Pedagógica, 11, 1.

Guerrero, F., \& Faro, T. (2012). Breve análisis del concepto de Educación Superior.

Granados, A. (2015). Las TIC en la enseñanza de los métodos numéricos. Sophia Educación, 11(2), 143-154

Alternativas en Psicología. Alternativas en Psicología, 16(27), 34-41.

Medeiros, D. A. (2001). Un estudio empíricoanalítico del uso de ordenadores en la enseñanza y aprendizaje.Murcia.
Edutec 2001, Actas del Congreso Internacional de Tecnología, Educación y Desarrollo Sostenible.

Mestres, L. (2008). La alfabetización digital de los docentes. [Educaweb. com] Recuperado de: http://www.educaweb. com/noticia/2008/12/01/ alfabetizaciondigital-docentes-3349/

Ong, W. (1987). Oralidad y escritura. Tecnologías de la palabra. México: Fondo de Cultura

Económica,

Rangel, P., \& Peñalosa, E. (2013). Alfabetización digital en docentes de educación superior: construcción y prueba empírica de un instrumento de evaluación. PixelBit. Revista de Medios y Educación, 43. Doi: https://doi.org/10.12795/ pixelbit.2013.i43.01

Tapia, E. \& Léon, J. (2013). Educación con tic para la sociedad del conocimiento. Revista Digital Universitaria [en línea], 14(2). Recuperado de http:// www.revista.unam.mx/vol.14/num2/ art16/\#up

Unesco (09 de octubre, 1998). Declaración mundial sobre la educación superior en el siglo XXI: Visión YAcción. Recuperado de: http://www. unesco.org/education/ educprog/wche/declaration_spa.htm

Valencia, H. G., Enriquez, J. A. V., \& Agredo, P. M. (2017). Strategies Used by Professors through Virtual Educational Platforms in Face-To-Face Classes: A View from the Chamilo Platform. English Language Teaching, 10(8).

Recuperado de: http://www. englishatuniversity.com/files/EL2.pdf 
Valencia, H. G., Valencia, A. R., \& Enriquezia, J. A. V. (2019). Integrating Technology In The Improvement And Motivation Of Learning English As A Foreign.

Recuprado de: http://www. englishatuniversity.com/files/INTER.pdf

Zamora, G. y Zerón A.M. (2010). Caracterización y sentido actual de la autoridad pedagógica en escuelas chilenas de sectores de pobreza. Revista Española de Pedagogía 68(245). 